\title{
A New Theoretical Approach to Location Based Power Aware Routing
}

\author{
Tarandeep Singh Kang ${ }^{1}$, Maninder Kaur ${ }^{2,}$ Dr K V P Singh ${ }^{3}$ \\ ${ }^{1}$ (ECE, Doaba Institute of Engineering \& Technology/Punjab Technical University, India) \\ ${ }^{2}$ (ECE, Doaba Institute of Engineering \& Technology/Punjab Technical University, India) \\ ${ }^{3}$ (ECE, Doaba Institute of Engineering \& Technology/Punjab Technical University, India)
}

\begin{abstract}
In MANETs, when information is sent from the source to the destination, these information packets travel through all the nodes which come across the path from the source to the destination. If the battery backup of any node across this path is less than the minimum time required to transmit the information packets from the source to the destination, then this information cannot be sent. In this proposed work, alternate methods are proposed to overcome this problem. One such proposed solution is to utilize Root up time Factor (RUF) for calculating the battery backup of all the nodes and then to use only those nodes which have maximum battery backup.
\end{abstract}

Keywords: Location based routing, Root up time factor, Bandwidth utilization.

\section{Introduction}

The increasing use of wireless portable devices such as mobile phones and laptops is leading to the possibility for spontaneous or ad hoc wireless communication known as Mobile Ad Hoc Networks (MANET). In contrary to infrastructure networks, an ad-hoc network lacks any infrastructure. There are no base stations, no fixed routers and no centralized administration. All nodes may move randomly and are connecting dynamically to each other. Therefore all nodes are operating as routers and need to be capable to discover and maintain routes to every other node in the network and to propagate packets accordingly. MANET may be used in areas with little or no communication infrastructure: think of emergency searches, rescue operations, or places where people wish to quickly share information, like meetings etc.

A mobile ad hoc network (MANET) is an autonomous system of mobile routers connected by wireless links. The union of which forms an arbitrary graph. The routers are free to move randomly and organize themselves arbitrarily; thus, the network's wireless topology may change rapidly and unpredictably. Such a network may operate in a stand-alone fashion, or may be connected to the larger Internet. In general, MANET is formed dynamically by a set of mobile nodes that are connected via wireless links without using an existing network infrastructure or centralized administration. These nodes are free to move randomly and organize themselves arbitrarily thus the topology of the network may change rapidly and unpredictably. MANET is an infrastructure-fewer network because it does not require any fixed infrastructure support such as a base station for its operation. Nodes participating in the mobile ad hoc networks manage routing without the use of existing infrastructure; these mobile nodes will typically have limited transmission range, which mean that packets might have to forward to another nodes to reach its destination.

\section{Routing Protocols Used in MANET's}

Network routing in MANETs can be classified into three classes, proactive, reactive, and hybrid. Routing schemes use control messages to learn the network topology. Most proactive routing schemes use link state or distance vector routing algorithms.

\section{Reactive Routing Protocols}

Reactive routing is an on-demand routing scheme. Nodes learn about the network topology on an asneeded basis. Two of the better known reactive ad hoc routing protocols are Dynamic Source Routing (DSR) and Ad hoc On-demand Distance Vector (AODV) . Reactive routing protocols are characterized by two phases, the route discovery phase and the route maintenance phase. In the route discovery phase, nodes build their routing tables on demand. The sending node sends a route request for a packet in its sending queue for which it has no route information to the destination or whenever a node receives a packet not destined to it for which it has no route information to the destination. Nodes send route inquiries to neighbours and add an entry to a list of previously discovered routes. For example, in AODV, nodes along the route store the route information, while in DSR the initiating node stores the source route returned to it from the route reply. The route maintenance phase uses route error packets and acknowledgments to maintain routes. Each node that sends a packet gets an acknowledgment back. Acknowledgments are either received at the link layer as defined by the MAC protocol 
of IEEE 802.11, or as passive acknowledgment from the receiving node. If the receiving node fails to acknowledge the receipt of the packet, a route error is relayed back to the original senders which results in the removal of the broken link from its cache and use an alternative route if available or initiate a new route discovery. Route discovery and route maintenance do consume bandwidth, however reactive routing is preferable over proactive in situations where route concentration, route activity, and the number of active routes per node is low and mobility is high.

\section{Proactive Routing Protocols}

In proactive routing, nodes periodically monitor the network for changes in network topology. Therefore, every node in the network keeps an up-to-date copy of current network topology information by periodically broadcasting and receiving control packets. For instance, when a node receives a packet destined to another node, it knows how and where to forward the packet for final delivery of the packet. This relatively detailed information about the topology helps to improve the routing performance. However, this improvement in routing may come at a cost of increased overhead and a decrease in network capacity for data. Several proactive routing exist for MANETs. Two commonly referenced ones are Optimized Link State Routing (OLSR) and Destination Sequenced Distance Vector Routing (DSDV). The way in which network topology information is gathered in proactive routing protocols is usually based on either of two algorithms, link state or distance vector.

\section{Link State Protocols}

In a link state protocol, each node keeps track of the changes that the network undergoes by keeping a map that reflects the state of all links in the network. Nodes use flooding, perhaps with some optimizations, to broadcast the link costs of their outgoing links to all neighbouring nodes .By exchanging link state, nodes learn the topology of the network. Each node creates a link-state packet that contains its identifier (ID), a list of its directly connected neighbours along with its cost to each one of them, a sequence number, and a time-to-live (TTL) value for the packet. These packets are broadcast whenever the TTL period expires or when the network topology changes. Link state routing is divided into two steps, flooding the network with link state information and the computation of routes based on link-state information using, typically, shortest path algorithm.

\section{Distance Vector Protocols}

Distance vector protocols are based on the assumption that each node knows the distance (cost) to its immediate neighbours. Thus, in distance vector protocols, all nodes create a list of distances to their immediate neighbours and distribute this list to their immediate neighbours. Initially, each node assigns a cost of one to each of its immediate neighbours and a cost of infinity to other nodes. Afterwards, the nodes exchange these lists with all of their immediate neighbours and start to replace the large costs with lower ones based on the received lists. Distance vector protocols suffer from several problems. The count-to18 infinity problem occurs when a node fails and, as such, its neighbours keep increasing their cost to reach this node until they reach an infinite value. Another problem with distance vector protocols is the slow convergence of routes throughout the network. By storing little information about links that are not directly connected to the node running the algorithm, distance vector protocols have lower complexity than link state protocols. In addition, the bandwidth requirement is also less.

\section{Hybrid Routing Protocols}

Hybrid routing protocols combine the use of reactive and proactive routing protocols to obtain a better balance between the dynamic nature of MANETs and the routing overhead and to reduce the average end-to-end delay. These protocols usually introduce a hierarchical structure to the MANET to reduce the number of control packet retransmissions during route discovery. In hybrid routing protocols, each node maintains a set of nearby neighbours with which it will use a proactive routing scheme and a set of more distant nodes with which it will use a reactive routing scheme. The use of different routing strategies at different times at different locations is used in routing protocols such as the Zone Routing Protocol (ZRP)

\section{Related Work}

Mobile ad hoc networks (MANETs) are characterized by dynamic topology, limited channel bandwidth and limited power at the nodes. Because of these characteristics, paths connecting to the source nodes with destinations may very unstable and go down at any time, making communication over ad hoc networks difficult. Energy efficiency is a limiting factor in the successful deployment of MANETs, because nodes are expected to rely on portable, limited power sources. Moreover, energy conservation is extremely challenging in multi-hop environments, where the mobile nodes should also consume energy to route packets for other 
Node position information in ad hoc networks will soon be obsolete due to its frequent movement [3]. The traditional location-based algorithms that assume the position of destination is reasonably accurate are not able to deal with eventual position deviation and impose high mobility tracking overhead. Moreover, once source node is ignorant of position of destination node, flooding scheme is used instead, which increases control overhead.

it theoretical analysis, by means of a cooperative relay, the probability of successful packet reception can be increased, and the overall power for routing can be reduced, given the outage probability of the link constrained at a certain target level. PLCR algorithm uses the location information of nodes to select the optimum next-hop node and cooperative node hop by hop with minimum power so that the cooperative route with minimum overall power from source to destination can be set up

\section{Proposed Routing Protocol}

The main objective of this proposed approach is to extend the network lifetime by improving the power utilization of the routing mechanism in MANETs. We utilize the ability of wireless network cards to dynamically change the transmission power, as well as bandwidth of the node. The ability of wireless devices is to read the remaining battery of the node and required bandwidth for communication between source to destination. Link stability are assigned according to the transmission power needed to reach the destination node, along with the bandwidth status of the sending and intermediary nodes. Our hypothesis is that choosing routes with lower uptime values on average with time and minimum required bandwidth node, will lead to better utilization of the power sources and bandwidth of the communicating devices.

In order to achieve the above objective, following outline is proposed:

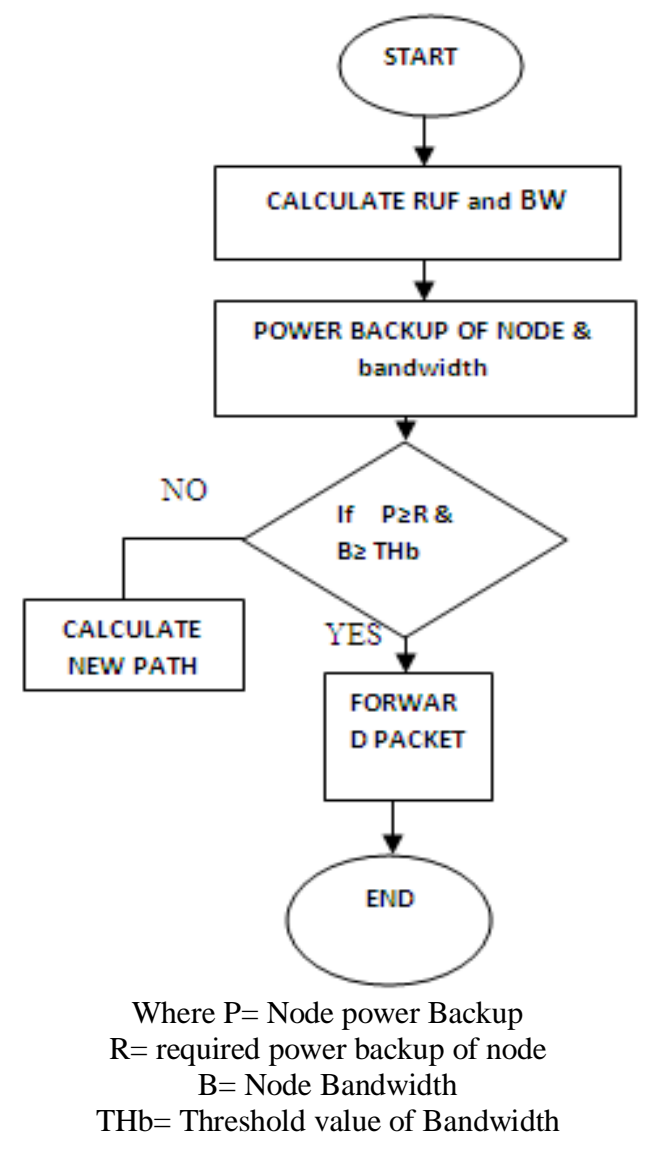

\section{Conclusion \& Future Work}

In this paper we have proposed a theoretical protocol to extend the network lifetime by improving the power utilization of the routing mechanism in MANETs. We utilize the ability of wireless network cards to dynamically change the transmission power, as well as bandwidth of the node. The ability of wireless devices is to read the remaining battery of the node and required bandwidth for communication between source to destination. Link stability is assigned according to the transmission power needed to reach the destination node, along with the bandwidth status of the sending and intermediary nodes. Our hypothesis is that choosing routes with lower uptime values on average with time and minimum required bandwidth node, will lead to better 
utilization of the power sources and bandwidth of the communicating devices. In future work this approach will be utilised to design a new protocol after proper verification and validation through all Location Based Routing Protocol. For validation of propose routing Protocol, we use Mat Lab to simulate the propose routing protocol and then compare with other Routing Protocol.

\section{References}

[1] B. P. E. U.-B. A. El Gamal, C. Nair and S. Zahedi. "Energy-efficient Scheduling of Packet Transmissions over Wireless Networks". IEEE INFOCOM, IEEE Proceedings of Twenty-First Annual Joint Conference of the IEEE Computer and Communications Societies, 3(2002):1773-1782, 2002.

[2] Ching-Wen Chen, Chuan-Chi Weng,"A Bandwidth-Based Routing Protocol with Efficient Path Discovery in Mobile Ad Hoc Networks", 2009 10th International Symposium on Pervasive Systems, Algorithms, and Networks

[3] Jangsu Lee, Seunghwan Y00, and Sungchun Kim," Energy aware Routing in Location based Ad-hoc Networks", Proceedings of the 4th International Symposium on Communications, Control and Signal Processing, ISCCSP 2010, Limassol, Cyprus, 3-5 March 2010

[4] Juanfei Shi, Kai Liu," Power-efficient Location-based Cooperative Routing in Wireless Networks",2012 International Conference on Systems and Informatics (ICSAI 2012)

[5] Mohammad A. Mikki," Energy Efficient Location Aided Routing Protocol for Wireless MANETs", (IJCSIS) International Journal of Computer Science and Information Security Vol. 4, No. 1 \& 2, 2009

[6] N. C. Wang, Y. F. Huang, J. S. Chen, S. M. Wang, C. L. Chen, "An Improved Location-Aided Routing Protocol for Mobile Ad Hoc Networks with Greedy Approach,” vol.8, Issue 8, Wseas Transaction on Communications,” 2009.

[7] Pramita M, Christian P and Shivajit M" Stability Aware Routing: Exploiting Transient Route Availability in MANETs Proceeding HPCC'07 Proceedings of the Third international conference on High Performance Computing and Communications Pages 508520, Springer-Verlag Berlin, Heidelberg (C2007

[8] Dr. P.K. Suri, Dr. M.K. Soni, Parul Tomar," Frame work for Location Based Power Aware Routing in MANET" IJCSI International Journal of Computer Science Issues, Vol. 8, Issue 3, May 2011.

[9] Sanzgiri, K., LaFlamme, D., Dahill, B., Levine, B.N., Shields, C. and Belding-Royer, E.M, "Authenticated Routing for Ad Hoc Networks", IEEE Journal on Selected Areas in Communications, Vol. 23, No. 3, Pp. 598-610,2005.

[10] T. F. Shih H.C. Yen, "Location-aware routing protocol with dynamic adaptation of request zone for mobile ad hoc networks", Springer Wireless Network 14:321-333, DOI 10.1007/s11276-006-9955-y, 2008. 